\title{
CORRECTION
}

\section{Correction to: Single lung transplantation for lymphangioleiomyomatosis: a single-center experience in Japan}

\author{
Hisashi Oishi ${ }^{1}(0) \cdot$ Tatsuaki Watanabe $^{1} \cdot$ Yasushi Matsuda $^{1} \cdot$ Masafumi Noda $^{1} \cdot$ Yutaka Ejima $^{2} \cdot$ Yoshikatsu Saiki $^{3}$. \\ Kuniaki Seyama ${ }^{4} \cdot$ Takashi Kondo $^{5} \cdot$ Yoshinori Okada $^{1}$
}

Published online: 13 June 2018

(c) Springer Nature Singapore Pte Ltd. 2018

\section{Correction to: Surgery Today https://doi.org/10.1007/s00595-018-1678-z}

In the original publication, Fig. 3 has been incorrectly published. The correct version of Fig. 3 is given in this correction.

The original article can be found online at https://doi.org/10.1007/ s00595-018-1678-z.

The original article has been corrected.

Hisashi Oishi

hisashi.oishi.c7@tohoku.ac.jp

1 Department of Thoracic Surgery, Institute of Development, Aging and Cancer, Tohoku University, 4-1 Seiryomachi, Aobaku, Sendai 980-8575, Japan

2 Department of Anesthesiology, Tohoku University Hospital, Sendai, Japan

3 Department of Cardiovascular Surgery, Tohoku University Graduate School of Medicine, Sendai, Japan

4 Division of Respiratory Medicine, Faculty of Medicine and Graduate School of Medicine, Juntendo University, Tokyo, Japan

5 Department of Thoracic Surgery, Tohoku Medical and Pharmaceutical University, Sendai, Japan

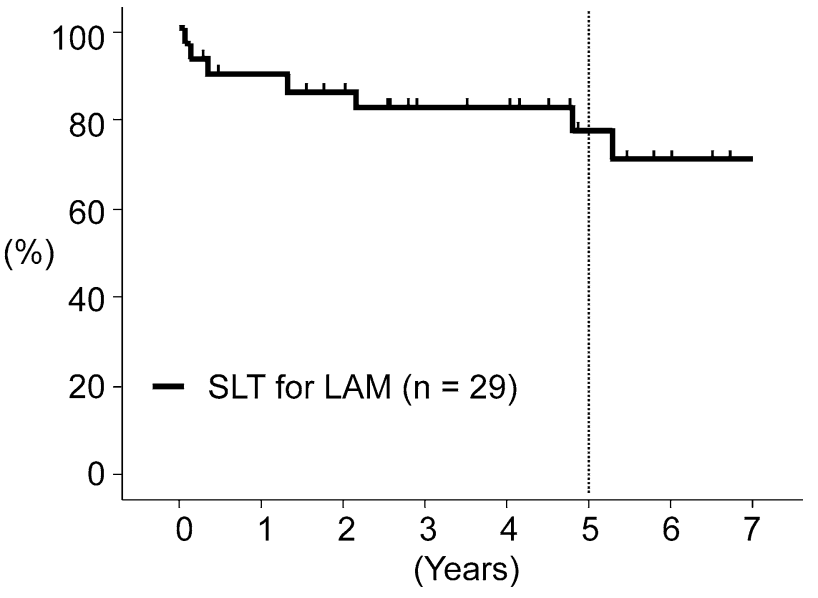

